OPEN ACCESS

Edited by:

Brigitte Mauch-Mani,

University of Neuchâtel, Switzerland

Reviewed by:

Zonghua Wang,

Fujian Agriculture and Forestry

University, China

Yun Chen,

Zhejiang University, China

${ }^{*}$ Correspondence:

Huiquan Liu

liuhuiquan@nwsuaf.edu.cn

Specialty section:

This article was submitted to

Plant Microbe Interactions,

a section of the journal

Frontiers in Plant Science

Received: 16 December 2016

Accepted: 23 January 2017

Published: 14 February 2017

Citation:

Wang Q, Jiang C, Wang C, Chen C,

$\mathrm{Xu} J$-R and Liu H (2017)

Characterization of the Two-Speed

Subgenomes of Fusarium graminearum Reveals the Fast-Speed Subgenome Specialized for Adaption and Infection. Front. Plant Sci. 8:140. doi: 10.3389/fpls.2017.00140

\section{Characterization of the Two-Speed Subgenomes of Fusarium graminearum Reveals the Fast-Speed Subgenome Specialized for Adaption and Infection}

\author{
Qinhu Wang ${ }^{1}$, Cong Jiang ${ }^{1}$, Chenfang Wang ${ }^{1}$, Changjun $C^{2} n^{2}$, Jin-Rong $X u^{1,3}$ and \\ Huiquan Liu'*
}

1 State Key Laboratory of Crop Stress Biology for Arid Areas and College of Plant Protection, Northwest A\&F University, Yangling, China, ${ }^{2}$ College of Plant Protection, Nanjing Agricultural University, Nanjing, China, ${ }^{3}$ Department of Botany and Plant Pathology, Purdue University, West Lafayette, IN, USA

Fusarium head blight, caused by Fusarium graminearum, is one of the most severe diseases on wheat and barley worldwide. Although the genomic data of several strains were published, the intragenomic variation of $F$. graminearum was not well characterized. Here, we sequenced three Chinese strains and conducted genomewide comparisons. Our data revealed that all the sequenced strains were distinct from each other and over 350 genes were functionally lost in each of them. Variants of each strain were unevenly distributed in a highly conserved pattern along the chromosomes, resulting in a conserved two-speed genome. The fast subgenome has a lower GC content, shorter gene length, and higher variation of exon numbers than the slow subgenome. Genes related to interaction and pathogenicity, under positive selection, and up-regulated in planta were all significantly enriched in the fast subgenome. Furthermore, we found that the fast subgenome coincided with facultative heterochromatin regions that were repressed in vegetative stage but activated during infection as measured by RNA-seq and ChIP-seq data, suggesting that the fast subgenome is epigenetically regulated. Taken together, our data demonstrated that F. graminearum has a highly conserved two-speed genome and the fast subgenome responsible for adaption and infection is under the control of heterochromatin.

Keywords: Fusarium head blight, genomic variation, two-speed genome, positive selection, heterochromatin

\section{INTRODUCTION}

Wheat is one of the most cultivated staple crops that feed the world. Fusarium head blight (FHB), caused by Fusarium graminearum, is a great threat to the yields and quality of wheat, barley, and maize that directly relates to the survival and heath of human beings (Goswami and Kistler, 2004). F. graminearum can also generate mycotoxins, including deoxynivalenol and zearalenone that contaminates the infested kernels, which are harmful to humans and livestock (D'Mello et al., 1999; Hussein and Brasel, 2001; Haggblom and Nordkvist, 2015). In China, FHB was first reported in 1936, and its recent epidemics occurred in 2003, 2008, 2010, and 2012 (Zhang et al., 2012; 
Mehta, 2014). In particular, the FHB outbreak in 2012 is extremely destructive and over one-third of the wheat growing areas were affected (Mehta, 2014).

Decoding the genome is the first step for understanding the whole machinery for fungi development, infection, and spreading. The whole genome sequencing of F. graminearum American strain $\mathrm{PH}-1$ revealed that the pathogen has very few repeats sequences and much more transcription factors and hydrolytic enzymes than other fungi (Cuomo et al., 2007). Intraspecies comparison of an American strain GZ3639, which was shotgun sequenced with only 0.4 -fold coverage, with the $\mathrm{PH}$ 1 revealed that the high single-nucleotide polymorphism (SNP) regions were correlated with pathogen specialization and mainly located in the telomeric or sub-telomeric regions (Cuomo et al., 2007). Comparative genomics of different species of Fusarium showed that the lineage-specific regions in the genome of Fusarium oxysporum were responsible for pathogenicity (Ma et al., 2010). Further studies also showed that the Fusarium pathogens have the bipartite (two-speed) genome architecture (Zhao et al., 2014; Sperschneider et al., 2015) as what happened in many other pathogens (Dong et al., 2015), such as oomycete pathogen Phytophthora infestans (Haas et al., 2009) and fungal pathogen Leptosphaeria maculans (Dong et al., 2015).

Fusarium graminearum is a worldwide pathogen, population analyses showed that even in a local region, the isolates of F. graminearum are very diverse (Qu et al., 2008; Yang et al., 2008; Zhang et al., 2012; Talas and McDonald, 2015; van der Lee et al., 2015). Besides the genome of strain PH-1 (Cuomo et al., 2007; King et al., 2015), recently, the resequencing data of three strains isolated from Australia (Gardiner et al., 2014), America and Canada (Walkowiak et al., 2015) were published. However, whether these strains have a different or conserved genomic variation remains largely unknown. Furthermore, no Chinese strains of F. graminearum were reported although great differentiations were existed (Qu et al., 2008; Yang et al., 2008; Zhang et al., 2012; van der Lee et al., 2015). We thus began our work by sequencing three Chinese strains isolated from main wheat growing regions to survey the genomic variations of F. graminearum. We performed comparative genomics with the previously reported strains to characterize the two-speed genome of F. graminearum. We also carried out RNA-seq analysis to determine the gene expression differences in the two subgenomes during plant infection. Moreover, we explored the differences of selection pressures and histone modification acting on the two subgenomes. Our study revealed that the two-speed genome of F. graminearum is highly conserved among different strains, and the fast-speed subgenome drove adaptive evolution and infection by heterochromatin regulation.

\section{RESULTS}

\section{Great Diversification of the Resequenced Genomes of $F$. graminearum}

We resequenced three F. graminearum strains, HN9-1, HN-Z6, and YL-1 (Figure 1A) that were isolated from the main wheat growing regions in China. Along with the recently resequenced
Australian strain CS3005 (Gardiner et al., 2014), Canadian strain FG1 (Walkowiak et al., 2015), and US strain FG2 (Walkowiak et al., 2015), we compared these six genomes to the reference genome of strain PH-1 isolated from Michigan, USA (Cuomo et al., 2007) (Supplementary Table S1). For each strain, about $95,157 \pm 17,471$ variants (Table 1) were called. In these variants, SNPs were the dominant genomic variations (94.3\%). Small insertion and deletion variations (INDELs) only account for a small proportion of the total variants (Table 1). On average, the F. graminearum genome has $2.5 \pm 0.4 \mathrm{SNPs}$ per $\mathrm{kb}$.

With the 10,304 SNPs detected in the 0.4-fold genome sequence of $F$. graminearum strain GZ3639 (Cuomo et al., 2007; King et al., 2015), we have a total of 275,641 SNP sites compared to PH-1. SNP based phylogenomic analysis revealed that the strains from one country were not clustered together. For examples, the Chinese strain HN9-1 is clustered with the two US strains PH-1 and GZ3639 (Figure 1A). The two nationwide FHB outbreak strains YL-1 and HN-Z6 of China are most closely related to Canadian strain FG1 and US strain FG2, respectively (Figure 1A). While the Australian strain CS3005 is distant from all the other strains we analyzed here (Figure 1A). Furthermore, the number of unique SNPs in the resequenced strains, ranged from 15,453 to 37,946 , is greater than any of the interaction numbers of the SNPs among FG1, FG2, CS3005, YL-1, HN91, and HN-Z6 (Figure 1B). These results consistent with the previous population studies that great genetic diversification exists in F. graminearum isolates (Qu et al., 2008; Yang et al., 2008; Zhang et al., 2012; Talas and McDonald, 2015; van der Lee et al., 2015).

\section{Over 350 Genes Were Functionally Lost in Each of the Resequenced Strains}

When being mapped onto different genomic features, most variants of the resequenced strains are in the intergenic (32.2\%) and coding regions (30.7\%) (Figure 1C, blue column). Another one-third of them are in the $5^{\prime}$ or $3^{\prime}$ untranslated regions (UTRs; Figure 1C, blue column). To remove the potential effects of total lengths on the proportion of different genomic features, we normalized the variants proportions by the relative sizes of different genomic features. We found that the intergenic regions have a high degree of variation; the UTRs and intron have a comparable variation density, both higher than the coding regions but lower than the intergenic regions (Figure 1C, red column). This result indicates that the coding regions of F. graminearum were under strong purifying selection.

For the variants in the coding regions, $41.0 \%$ of them are missense variations. Remarkably, relative to the genome sequence of strain PH-1, a total of 4,994 variants (Table 2) caused start codon lost, stop codon gained, or frameshift, which potentially result in gene function losses. These variations affect 1,647 protein-coding genes, and for each strain, over 350 genes were functionally lost (Figure 1D). Among them, 41 genes were lost in all resequenced strains, while over 90 genes were specifically lost in each of the resequenced strains (Supplementary Figure S1). In addition, we identified 381 variants resulting in stop codon lost (Table 2) in the six resequenced strains that may affect translation 

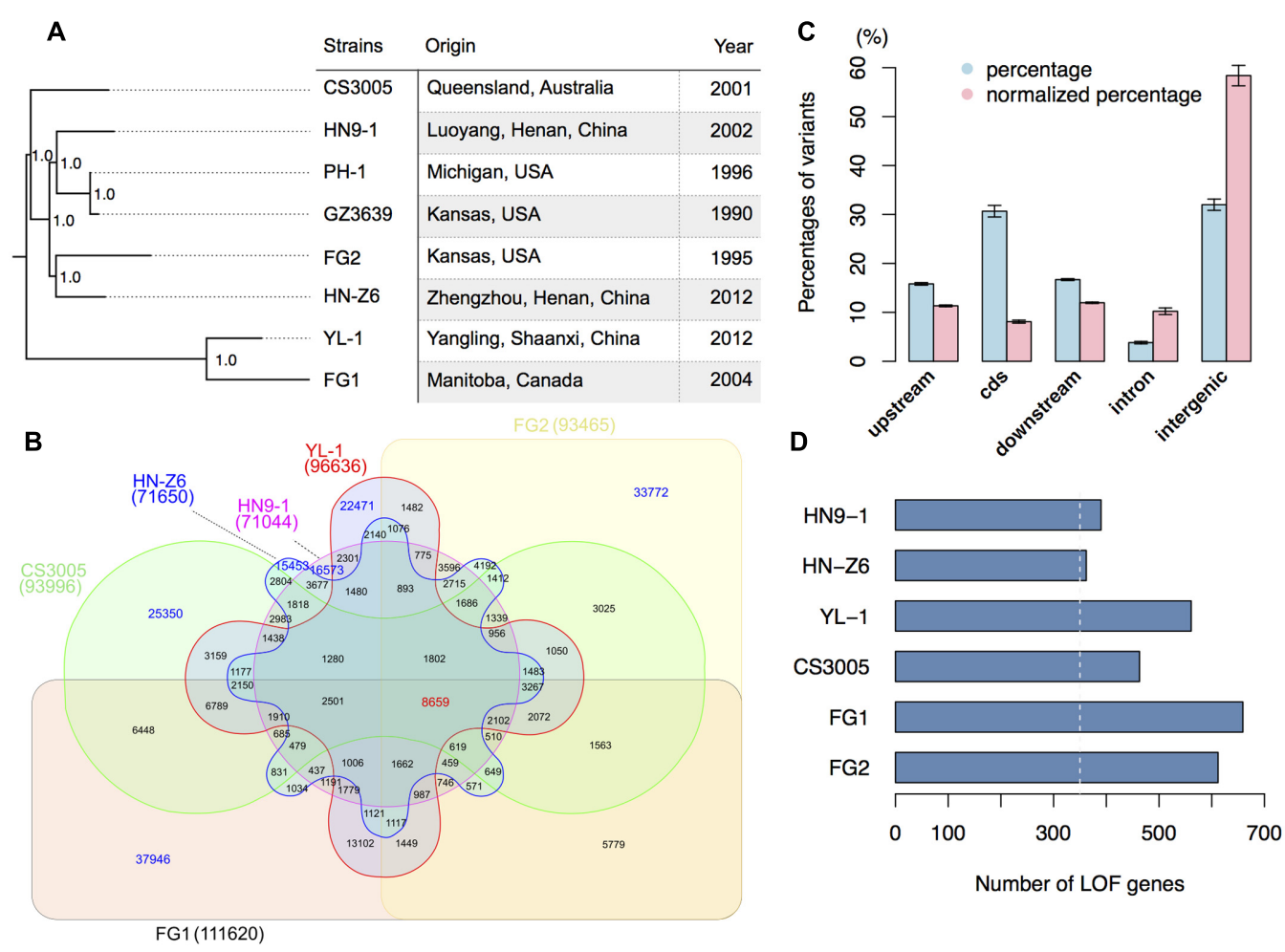

FIGURE 1 | Genomic variation of $\boldsymbol{F}$ graminearum strains. (A) Strains used in this study and their maximum likelihood phylogenetic tree based on SNP data. The tree was mid-point rooted. Numbers near the nodes indicate the branch support values ("1.0" means 100\%) generated by SH-like approximate likelihood ratio test (SH-aLRT). (B) Venn diagram showing the common and specific SNPs among different strains in comparison with the reference genome sequence of PH-1. Digit in the brackets indicates the total number of SNPs for each strain. (C) Bar chart showing the proportions of variants located in different genomic regions. The percentages of variants in different genomic regions are in blue, while the percentages of variants in different genomic regions normalized by the total length of the corresponding regions are in red. The upstream and downstream sequences (represent UTRs) are the 500-bp $5^{\prime}$ and $3^{\prime}$ flanking sequences of the coding region (cds) of individual genes. Error bars represent the standard deviations of the proportions among the six strains. (D) Number of genes that have predicted (in blue) loss-of-function (LOF) variants in the six strains. The white-dashed line marks 350.

TABLE 1 | Number of genomic variants identified in $\mathbf{F}$. graminearum strains.

\begin{tabular}{lccc}
\hline Strains & SNP & INDEL & Total variants \\
\hline HN9-1 & 71,044 & 3,598 & 74,642 \\
HN-Z6 & 71,650 & 3,582 & 75,232 \\
YL-1 & 96,636 & 5,436 & 102,072 \\
CS3005 & 93,996 & 5,136 & 99,132 \\
FG1 & 111,620 & 8,512 & 120,132 \\
FG2 & 93,465 & 6,265 & 99,730 \\
GZ3639 & 10,304 & - & - \\
\hline
\end{tabular}

and protein functions by adding a stretch of extra peptides to the C-terminal end.

\section{Fusarium graminearum Has a Highly Conserved Two-Speed Genome}

To study the distributions of genomic variations, we calculated the variant frequencies in different chromosomal regions. For all sequenced strains, the variants were unevenly distributed along the four chromosomes (Figure 2). The variants were
TABLE 2 | Number of variants that potentially result in gene loss-of-function.

\begin{tabular}{lcccc}
\hline Strains & Start lost & Stop gained & Frameshift & Stop lost \\
\hline HN9-1 & 38 & 199 & 299 & 47 \\
HN-Z6 & 29 & 180 & 287 & 46 \\
YL-1 & 54 & 301 & 416 & 66 \\
CS3005 & 40 & 272 & 360 & 65 \\
FG1 & 56 & 346 & 842 & 84 \\
FG2 & 40 & 333 & 902 & 73 \\
\hline
\end{tabular}

often enriched in specific chromosomal regions, especially in the telomeric or sub-telomeric regions (Figure 2). And this is consistent with the previous genome comparison between two American strains PH-1 and GZ3639 (Cuomo et al., 2007), and the inter-species comparison among Fusarium pathogens (Zhao et al., 2014; Sperschneider et al., 2015). Interestingly, the distribution patterns of variants were well conserved across all these strains (Figure 2), even in the specific SNP regions of each strain (Supplementary Figure S2). 


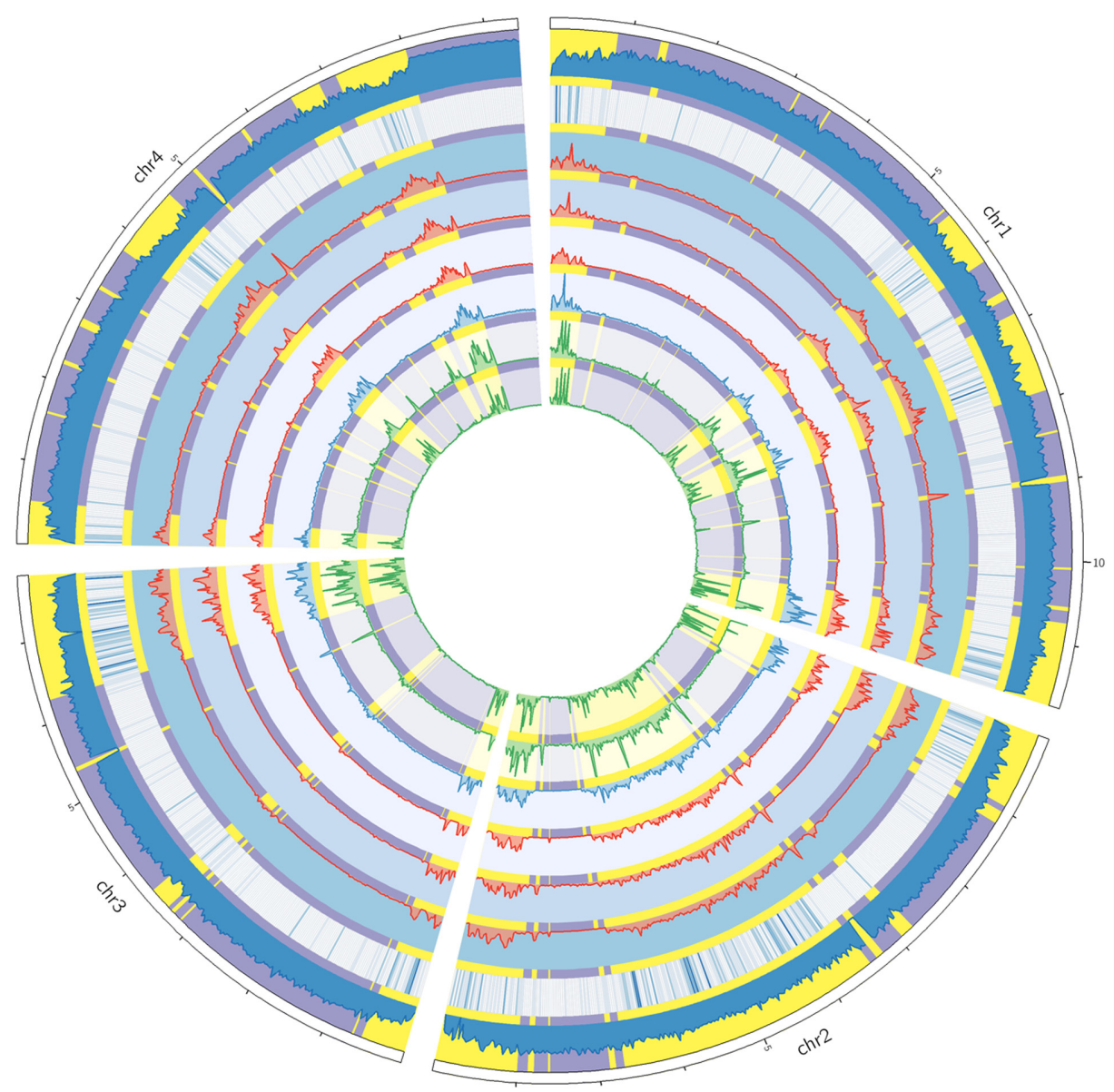

FIGURE 2 | Circos plot showing the variant distribution and conserved two-speed genome. From the circus outside to inside are the four chromosomes of F. graminearum, histogram of GC contents, heat map of secreted protein genes, and variation densities of strains YL-1 (in red), HN9-1 (in red), HN-Z6 (in red), CS3005 (in blue), FG1 (in green), and FG2 (in green). The fast subgenome regions (highlighted in yellow) and the slow subgenome regions (highlighted in purple) are calculated by depmixS4.

To analyze the variant distribution patterns in $F$. graminearum, we modeled the frequencies of variants (defined as number of variants per $\mathrm{kb}$ ) with the expectationmaximization algorithm (Benaglia et al., 2009) by using all the variants identified in the six well-sequenced strains. The results clearly showed that the genome could be divided into two subgenomes: a fast subgenome with high frequency of variants and a slow subgenome with low frequency of variants (Figure 3A), similar to previous results of interspecies genome comparison (Zhao et al., 2014; Sperschneider et al., 2015). The rates for the fast and slow subgenomes were estimated at $4.9 \pm 3.5$ and $0.6 \pm 0.3$ variants per $\mathrm{kb}$, respectively (Figure 3A). We used Viterbi algorithm (Visser and Speekenbrink, 2010) to determine the exact regions of the fast and slow speed subgenomes in F. graminearum (Supplementary Table S2). The results revealed that the fast subgenome contains 6,353 genes in $15.3 \mathrm{Mb}$ region (Figure 2, highlighted in yellow), and the slow subgenome contains 7,811 genes in $22.7 \mathrm{Mb}$ region (Figure 2, highlighted in purple).

\section{The Two Subgenomes of $F$. graminearum Have Distinct GC Content but Similar Gene Density}

To investigate the differences between the two subgenomes, we calculated the GC content in each interval of the two subgenomes. Intriguingly, the fast subgenome has a GC content of $46.8 \%$, which is significantly ( $p$-value $=7.9 \mathrm{e}-31$ ) lower than the slow subgenome (49.1\%) (Figures 2 and 3B). Consistent with this observation, the GC content is lower for genes located in the fast subgenome (50.5\%) than those in the slow subgenome (52.2\%) $(p$-value $=4.0 \mathrm{e}-207$, Supplementary Figure S3A). In addition, the gene length in fast subgenome region is slight shorter $(p$-value $=7.0 \mathrm{e}-3)$ than that in the slow subgenome region (Supplementary Figure S3B), and the exon number variation in the fast subgenome is much higher $(p$-value $=0)$ than that in the slow subgenome (Supplementary Figure S3C). We found that there are 415.2 and 344.1 genes per $\mathrm{Mb}$ in the fast and slow subgenomes, respectively. Thus the fast subgenome in F. graminearum does not coincide with the gene sparse region 

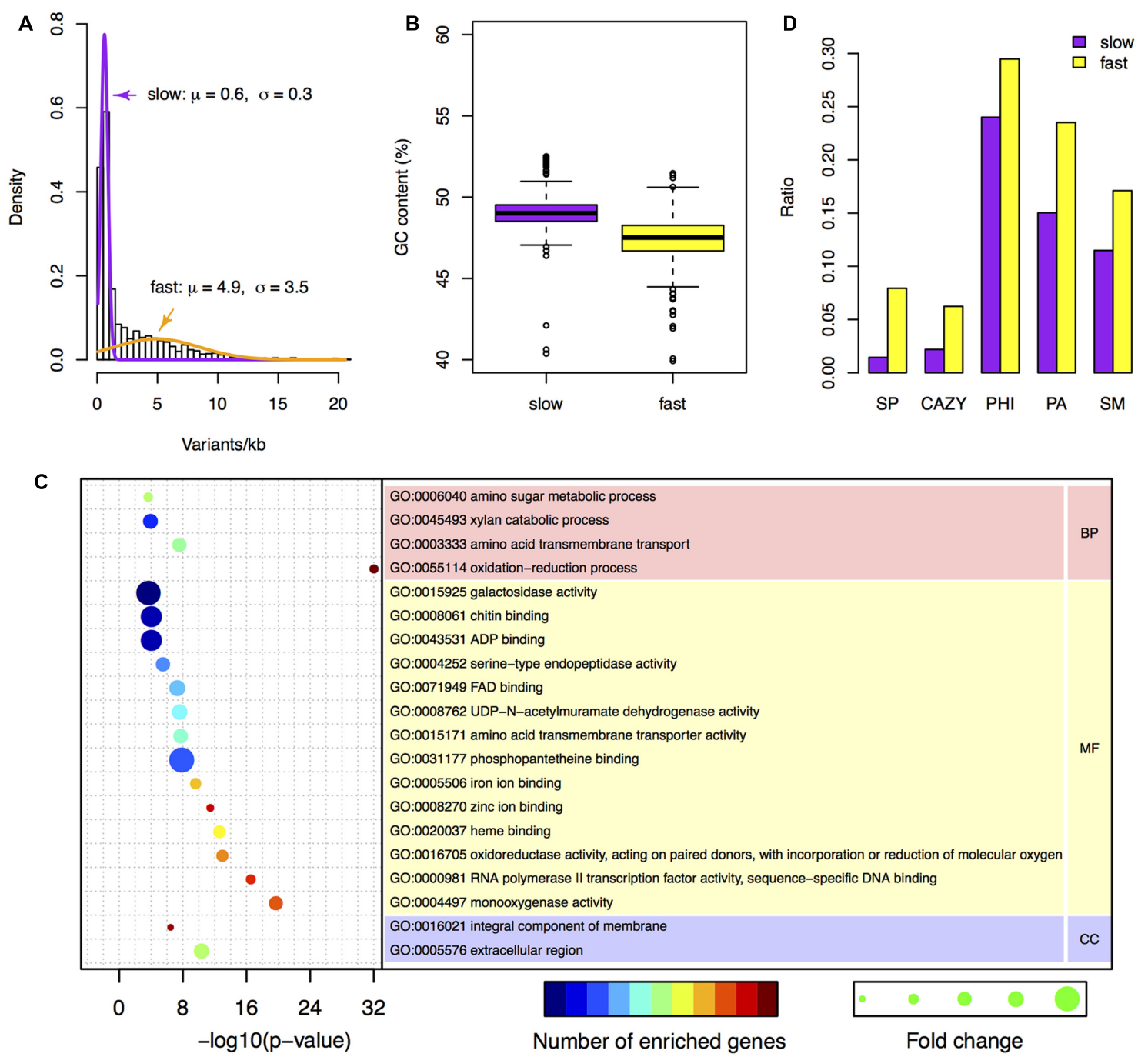

FIGURE 3 | Characteristics of the two-speed genome of $\boldsymbol{F}$. graminearum. (A) The probability density of the number of variants per kb among F. graminearum strains. The average numbers of variants per $25 \mathrm{~kb}$ in the six resequenced strains were used for modeling. Purple and orange curves represent the estimated probability distributions of the variants number for the slow and fast subgenome, respectively. (B) Boxplot showing the GC content of the fast and slow subgenomes. (C) GO enrichment analysis with the genes located in the fast subgenome of $F$. graminearum. BP, MF, and CC stand for biological process, molecular function, and cellular component, respectively. (D) The proportions of carbohydrate-active enzymes (CAZY) genes, pathogen-host interactions (PHI) genes, pathogen-associated (PA) genes, secondary metabolite (SM) genes, and secreted protein (SP) genes in the fast and slow subgenomes.

as what has been reported in repeat-rich oomycete pathogen P. infestans (Haas et al., 2009). Consistently, the gene borders, which consist of $5^{\prime}$ and $3^{\prime}$ flanking intergenic regions (FIRs), have a similar distribution in the fast and slow subgenomes of F. graminearum, which is also different with the two subgenomes of P. infestans (Haas et al., 2009; Supplementary Figure S4) and fungal pathogen L. maculans (Dong et al., 2015).

\section{The Fast Subgenome of $F$. graminearum Is Enriched for Genes Related to Interaction and Pathogenicity}

To study the function of the fast subgenome, we performed gene ontology (GO) enrichment analysis. The fast subgenome is enriched for genes involving in xylan catabolic, amino acid transmembrane transport, amino sugar metabolic, and oxidation-reduction processes (Figure $3 \mathrm{C}$ ). The proteins located in extracellular region were also overrepresented, suggesting that the fast subgenome may associate with the secreted proteins (SP). We thus compared the composition of SP genes in the two subgenomes. The completed $F$. graminearum genome has a total of 616 genes that encode SP (Cuomo et al., 2007). Of these, $504(81.8 \%)$ are located in the fast subgenome, whereas only $112(18.2 \%)$ are located in the slow subgenome. Thus, the fast subgenome is significantly $(504 / 6353 \div 112 / 7811=5.5-$ fold, $p$-value $=5.5 \mathrm{e}-83$ ) enriched for SP genes (Figures 2 and 3D). Consistent with this result, the carbohydrate-active enzymes (CAZY; Henrissat and Davies, 1997) that required for 
the degradation of plant cell wall to facilitate infection and/or gain nutrition were 2.8-fold enriched in the fast subgenome (Figure 3D). Furthermore, we found that the known pathogenhost interactions (PHI) genes (Urban et al., 2015), pathogenassociated (PA) genes (Sperschneider et al., 2013), and secondary metabolite (SM) genes (Sieber et al., 2014) are all overrepresented in the fast-speed subgenome (Figure 3D). These results suggest that the fast-speed subgenome of F. graminearum is enriched for interaction and pathogenicity related genes.

\section{The Fast Subgenome of $\boldsymbol{F}$. graminearum Is Enriched for Genes under Positive Selection}

To determine whether genes in the two subgenomes are underwent different selection pressures, we calculated the number of synonymous differences per synonymous site (pS) and the number of non-synonymous differences per nonsynonymous site $(\mathrm{pN})$ for individual F. graminearum genes with SNPs. By using two different methods (see Materials and Methods), a total of 1181 candidate of positive selected genes were found (Figures 4A,B). Among them, 609 genes (9.6\%) are in the fast subgenome, which is 1.3 -fold higher $(p$-value $=7.9 \mathrm{e}-7)$ than the rest 572 genes $(7.3 \%)$ in the slow subgenome.

Additionally, the non-synonymous difference value $\mathrm{pN}$ in the fast subgenome is much higher $(p$-value $=4.8 \mathrm{e}-47)$ than that in the slow subgenome (Figure 4C). Furthermore, we found that the PA and SM genes in the fast subgenome contained more positively selected genes compared to that in the slow subgenome (Figure 4D), when normalized to the number of PA or SM genes in the each subgenomes. Positive selection can relate to different adaptation processes, such as environmental, geographical, host response. Therefore, our data further suggest that the fast subgenome of F. graminearum is enriched for genes required for adaptation.

\section{The Fast Subgenome of $\boldsymbol{F}$. graminearum Is Enriched for Genes Up-Regulated In planta}

To determine differences in the gene expressions in the fast and slow subgenomes during infection, we sequenced the samples of 6 days post-inoculated wheat heads, and performed RNA-seq analysis to find the differently expressed genes (DEGs) in planta. Of the 14,164 reference genes, 10,853 (76.6\%) have at least one count per million in each of the two biological repeats. By using the gene expression data of mycelia as a background, a total of 4,737 genes (33.4\%) were found to be differently expressed during plant infection, including 2,243 genes (15.8\%) up-regulated and 2,494 genes (17.6\%) down-regulated over two folds (Table 3; Figure 5A).

About 36.2\% genes (2,297 genes) are differently expressed in the fast subgenome, which is significantly higher than the $31.2 \%$ DEGs (2,440 genes) in the slow subgenome (Table 3). Interestingly, this difference is absolutely caused by the upregulation of genes in the fast subgenome, since the genes down-regulated have no significant differences $(p$-value $=0.48)$ between the two subgenomes (Table 3; Figure 5B). There are $1177(18.5 \%)$ and 1066 (13.6\%) up-regulated genes in the fast and slow subgenome, respectively (Table 3; Figure 5B). Moreover, the number of extremely up-regulated genes $(\geq 10$ fold) in the fast subgenome is 4.8 -fold enrichment than that in the slow subgenome (Figure 5B). Notably, the extent of gene up-regulation and down-regulation is much higher (1.8fold with $p$-value $=8.5 \mathrm{e}$-92 for up-regulated DEGs, 1.3 -fold with $p$-value $=7.3 \mathrm{e}-28$ for down-regulated DEGs) in the fast subgenome than in the slow subgenome (Figure 5C), indicating that the fast subgenome may contains more genes that are involved in rapid response to host plants. Notably, the SP, CAZY, PHI, PA, and SM genes in the fast subgenome contained more genes up-regulated in planta compared to that in the slow subgenome (Figure 5D). All these data suggest that the fast subgenome of F. graminearum play important roles for plant infection.

\section{The Fast Subgenome of $F$. graminearum Is Associated with Facultative Heterochromatin}

It have been recognized that the regulation of massive concerted expression of pathogen genes during infection is chromatinbased, and these genes are located in the low GC isochores (Soyer et al., 2015). Hence, the rapid up-regulation of the genes in the fast subgenome is likely epigenetically programmed by chromatin. To verify if the fast subgenome is associated with the plastic heterochromatin in F. graminearum, we mapped the facultative heterochromatin mark H3K27me3 associated ChIPseq reads generated by Connolly et al. (2013) to the genome. Interestingly, the fast subgenome is overwhelmingly located in the facultative heterochromatin regions with high level of H3K27me3 marks (Figure 6, red track, highlighted in yellow). In contrast, the activation marks (H3K4me2, H3K4me3) are co-localized with the slow subgenome (Figure 6, green tracks). Therefore, the fast subgenome is correlated with the facultative heterochromatin represented by the repressive H3K27me3 mark, and the slow subgenome is correlated with the euchromatin represented by the activating $\mathrm{H} 3 \mathrm{~K} 4 \mathrm{me} 2 / \mathrm{H} 3 \mathrm{~K} 4 \mathrm{me} 3$ mark. All these observed correlations were further verified by Spearman correlation analysis (Figure 6, inner correlation heatmap).

\section{Identification of Candidate Genes Responsible for Fusarium-Wheat Interaction}

To define genes responsible for Fusarium-wheat interaction, we considered the three points following. First, to battle with plant, a gene involved in infection directly is likely to be up-regulated. Second, if a gene is really important for pathogenicity, during the co-evolution of host and pathogen, it should bear with significant positive selection. Third, some of the genes (such as effector genes) involved in infection may secrete to the extracellular space or enter plant cell to facilitate infection. By applying these criteria, eight genes responsible for Fusarium-wheat interaction were identified (Figure 7; Table 4). Interestingly, six of these eight genes are in the fast subgenome while only two belong to the slow 


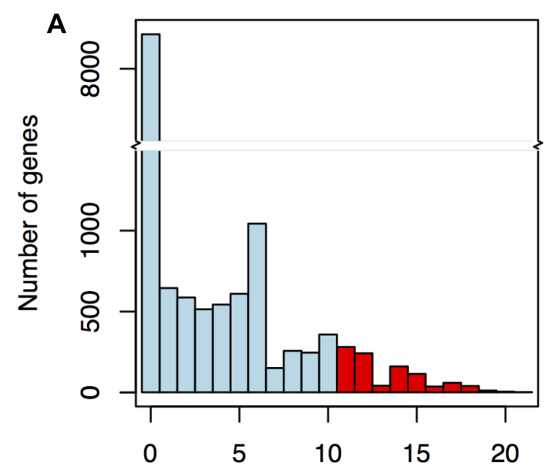

No. of the ortholog pairs with $\mathrm{pN}-\mathrm{pS}>0$

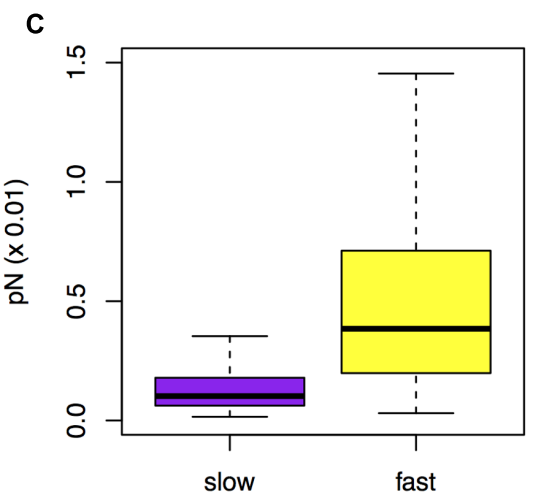

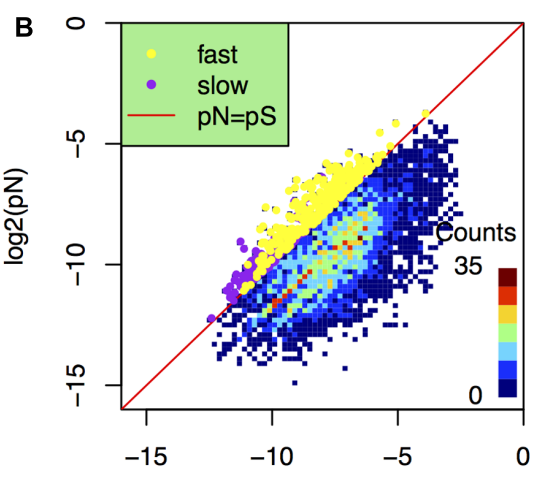

$\log 2(\mathrm{ps})$

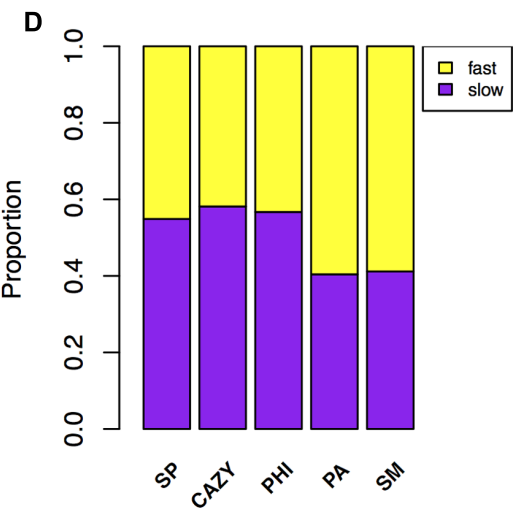

FIGURE 4 | Comparison of positive selection in the fast and slow subgenomes. (A) Histogram showing the number of genes in the categories that grouped by the total number of ortholog pairs with $\mathrm{pN}-\mathrm{pS}>0$. The red columns indicate the positive selected genes (screened by $\mathrm{pN}>\mathrm{pS}$ in at least 11 ortholog pairs). (B) The distribution of the positively selected genes (screened by the average pN/pS $>1$ and the average pS $>0$ ) in the fast and slow subgenomes. The $x$-axis and $y$-axis are the logarithm of pS and pN, respectively. (C) Comparison of non-synonymous rates (Langmead et al., 2009) in the fast and slow subgenomes. (D) The proportions of carbohydrate-active enzymes (CAZY) genes, pathogen-host interactions (PHI) genes, pathogen-associated (PA) genes, secondary metabolite (SM) genes, and secreted protein (SP) genes that underwent positive selection relative to the total number of them located in the fast or slow subgenome.

subgenome. In these genes, five of them have PHI database hits, including three genes can result reduced pathogen virulence upon interruption (Table 4), and one PA gene (Sperschneider et al., 2013) defined previously (Table 4). Interestingly, two of the candidates (FGRRES_10712 and FGRRES_15917_M) have a high sequence similarity with the effector candidates identified by interspecies comparison previously (Sperschneider et al., 2015), including a homolog of FGRRES_10999, which have underwent diversifying selection among species, but no SNPs were found within the eight strains analyzed in this study. Therefore, our intraspecies comparison has not only extended the effector candidates, but also supplied good candidates for recently active effectors.

\section{DISCUSSION}

Previous studies have shown that $F$. graminearum has a two-speed genome (Zhao et al., 2014; Sperschneider et al., 2015) and the highly variable regions are responsible for pathogen specialization (Cuomo et al., 2007). However, it is not clear whether the highly variable regions are conserved
TABLE 3 | The number of DEGs during plant infection for strain PH-1.

\begin{tabular}{lcccc}
\hline DEGs & $\begin{array}{c}\text { Fast } \\
\text { subgenome } \\
(\mathbf{6}, \mathbf{3 5 3} \text { genes) }\end{array}$ & $\begin{array}{c}\text { Slow } \\
\text { subgenome } \\
\mathbf{( 7 , 8 1 1} \text { genes) }\end{array}$ & p-value & Total \\
\hline Up-regulated & $1,177(18.5 \%)$ & $1,066(13.6 \%)$ & $1.8 \mathrm{e}-15$ & 2,243 \\
Down-regulated & $1,120(17.6 \%)$ & $1,374(17.6 \%)$ & 0.48 & 2,494 \\
Total & $2,297(36.2 \%)$ & $2,440(31.2 \%)$ & $4.0 \mathrm{e}-10$ & 4,737 \\
\hline
\end{tabular}

among different $F$. graminearum strains. In this study, we resequenced three Chinese strains of $F$. graminearum and performed comparative genomics of them with three American strains (Cuomo et al., 2007; Walkowiak et al., 2015), one Canadian strain (Walkowiak et al., 2015), and one Australian strain (Gardiner et al., 2014). Although great divergences exist in different strains, our data showed that the two-speed genome of F. graminearum is highly conserved among different strains. The fast subgenome has a lower GC content than the slow subgenome. All the interaction and pathogenicity related genes, including SP, CAZY, PHI, PA, and SM genes, were overrepresented in the fast subgenome. In addition, genes underwent positive 

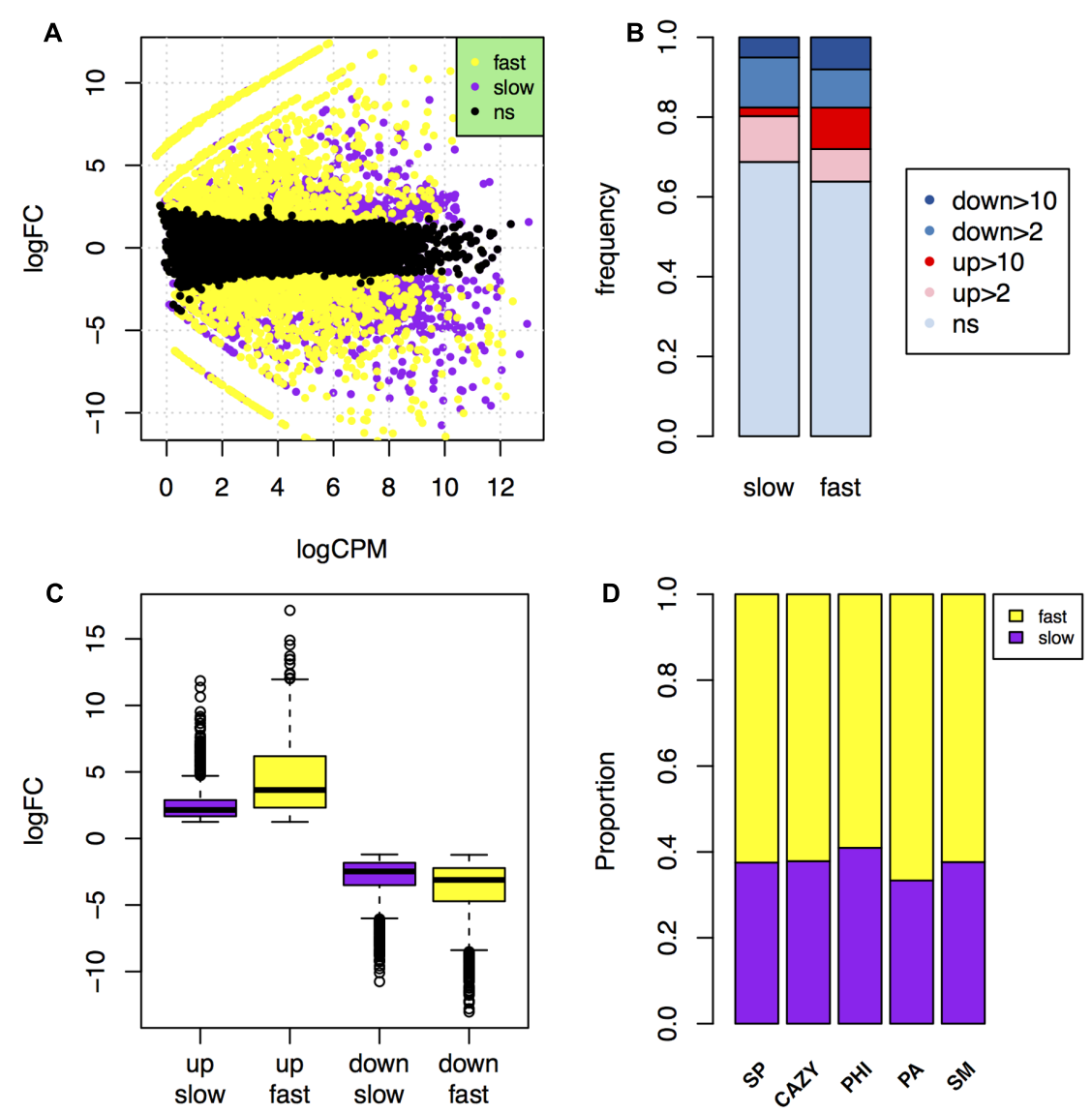

FIGURE 5 | Comparison of in planta gene expression in the fast and slow subgenomes. (A) MA-plot of genes that were up- or down-regulated over 2-fold during plant infection in the fast (yellow) and slow (purple) subgenomes. (B) Comparison of the frequency of genes up- or down-regulated over 2- or 10-fold in planta in the fast and slow subgenomes. ns, not significant. (C) Comparison of the fold change levels of the up-and down-regulated genes in the fast and slow subgenomes. (D) The proportions of carbohydrate-active enzymes (CAZY) genes, pathogen-host interactions (PHI) genes, pathogen-associated (PA) genes, secondary metabolite (SM) genes, and secreted protein (SP) genes that in planta up-regulated relative to the total number of them located in the fast or slow subgenome.

selection and/or up-regulated in planta were also enriched in the fast subgenomes of F. graminearum. These results suggest that the fast subgenome drives adaptive evolution and infection in F. graminearum.

We showed that the features of the two-speed genome of F. graminearum are different from that of fungal pathogen L. maculans (Grandaubert et al., 2014) and oomycete pathogen P. infestans (Haas et al., 2009). First, the two subgenomes of F. graminearum have a comparable subgenome sizes and number of genes, while the two subgenomes of L. maculans (Grandaubert et al., 2014) and P. infestans (Haas et al., 2009) are very different. Second, the fast subgenomes of L. maculans and P. infestans (Haas et al., 2009; Grandaubert et al., 2014; Dong et al., 2015) were coincided with the gene sparse regions. In contrast, the gene density in the fast subgenome of F. graminearum is slightly higher than the slow subgenome in F. graminearum. Furthermore, the fast subgenome has a slightly shorter gene length with larger exon number variation than the slow subgenome. Thus, the fast subgenome of F. graminearum may be a little bit more compact than the slow subgenome. These differences maybe due to very little of repetitive sequences in F. graminearum genome (Cuomo et al., 2007; King et al., 2015).

The distinct two-speed genome model of F. graminearum described here indicates that, although the diverse eukaryotic pathogens may have different origins for the fast subgenome, they are convergently evolved a subgenome for adaptive selection. The classical two-speed genome of plant pathogens is driven by repetitive sequences (Raffaele et al., 2010; Grandaubert et al., 2014; Dong et al., 2015). For now, it is not clear about the potential mechanism of two-speed genome evolved in F. graminearum. However, one common feature of the fast subgenomes in F. graminearum, L. maculans, and P. infestans is their AT-rich characteristic. Chromatin with high AT isochores (Soyer et al., 2015) is proposed as a concise and parsimony approach for the concerted expression of infection-related genes. In addition, the non-conserved region (relevant to the fast subgenome) of $F$. graminearum genome has weak gene expression in mycelia but has a highly variable gene expression 


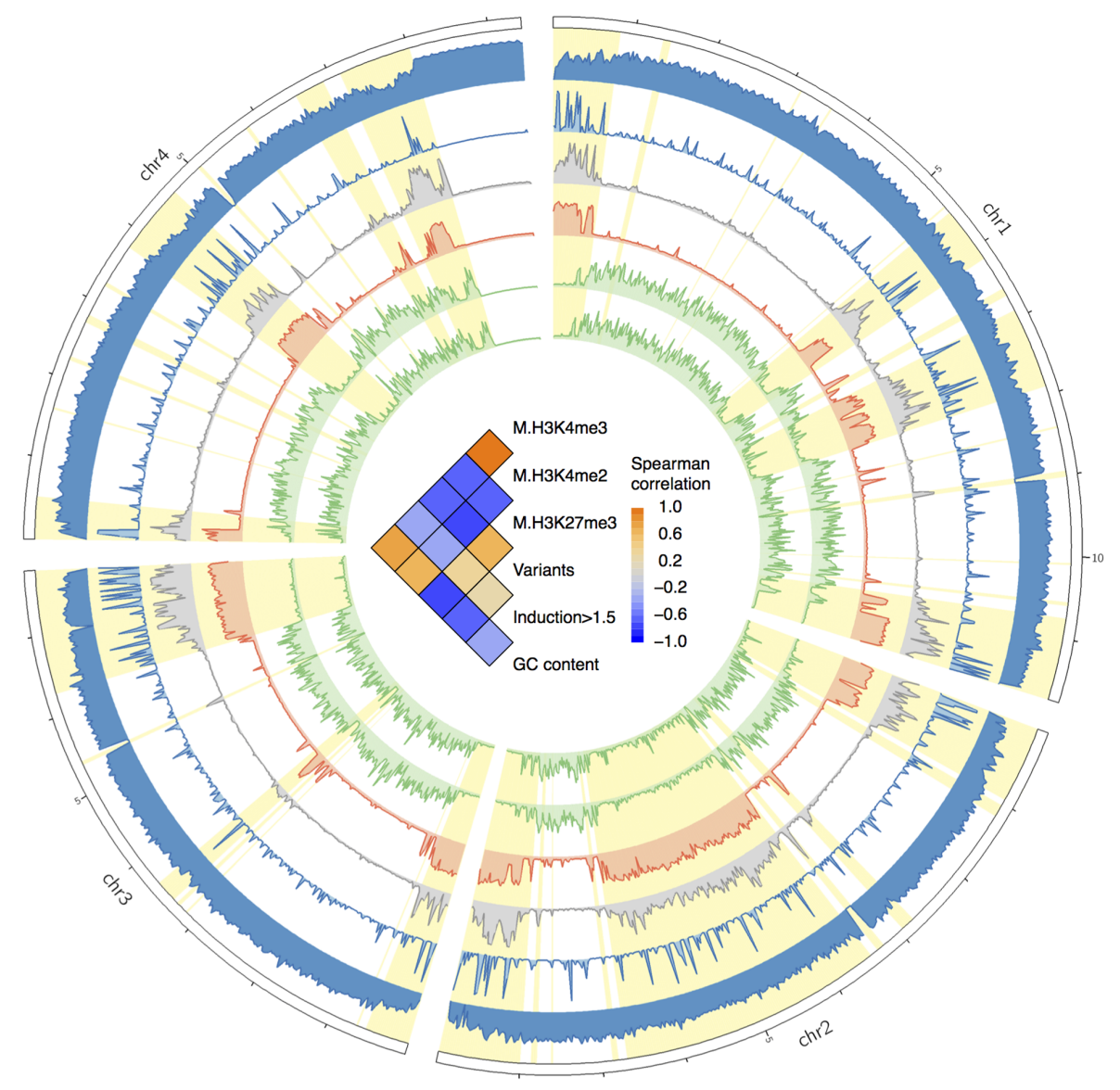

FIGURE 6 | Circos plot showing the distributions of GC contents, variants, genes upregulated in planta, and histone marks. From the circus outside to inside are the histogram of GC contents, gene expression fold changes in planta, variants frequency, and frequencies of histone mark of $\mathrm{H} 3 \mathrm{~K} 27 \mathrm{me} 3$, $\mathrm{H} 3 \mathrm{~K} 4 \mathrm{me} 2$, and H3K4me3. The fast subgenome region is highlighted in yellow. In the center of the circus, the heat-matrix shows the Spearman correlation coefficients between different tracks.

between mycelia and conidia stages (Zhao et al., 2014). In this study, we further demonstrated that the fast subgenome has higher variable gene expression in planta and in vitro. Therefore, the true difference under the two subgenomes may be lying on the chromatin structures.

We analyzed the ChIP-seq data generated before (Connolly et al., 2013), and the result showed a perfect correlation between $\mathrm{H} 3 \mathrm{~K} 27 \mathrm{me} 3$ and the fast subgenome. Thus the fast subgenome is the heterochromatin in hyphae stage, and $\mathrm{H} 3 \mathrm{~K} 27 \mathrm{me} 3$ is likely to be the silencing mark. Once infection to be or being established, the fast subgenome is reprogrammed to facility the rapid responses of infection and adaptation. In fact, heterochromatin is shown to regulate secondary metabolism genes and effectors in fungal pathogens F. graminearum, Fusarium verticillioides, and L. maculans (Reyes-Dominguez et al., 2012; Visentin et al., 2012; Soyer et al., 2014, 2015). Our study also showed that the fast subgenome is enriched for interaction and infection related genes, positive selected genes, and secondary metabolism genes, thus heterochromatin-based regulation of the fast subgenome is required for the infection and adaptation in F. graminearum.

\section{MATERIALS AND METHODS}

\section{Fungal Strains and Growth Condition}

Fusarium graminearum strains HN9-1 (kindly provided by Dr. Zhonghua Ma, Zhejiang University), HN-Z6 (kindly provided by Dr. Wenming Zheng, Henan Agricultural University), and YL-1 (this study) isolated from wheat head with FHB in China were maintained on the potato dextrose agar medium at $25^{\circ} \mathrm{C}$. The strains (available upon request) were deposited at the Northwest A\&F University - Purdue University Joint Research Center.

\section{Genome Resequencing Analysis}

For the Chinese strains HN9-1, HN-Z6, and YL-1, paired ends 90-bp high throughput DNA sequencing (Supplementary Table S1) was performed at Beijing Genomics Institution (Shenzhen, China). The resequencing data were deposited at NCBI SRA database under accession number SRP063887. The raw reads of Australian strain CS3005 (Gardiner et al., 2014) were downloaded from NCBI SRA database. The reads were mapped on the reference genome of strain PH-1 (King et al., 2015) 


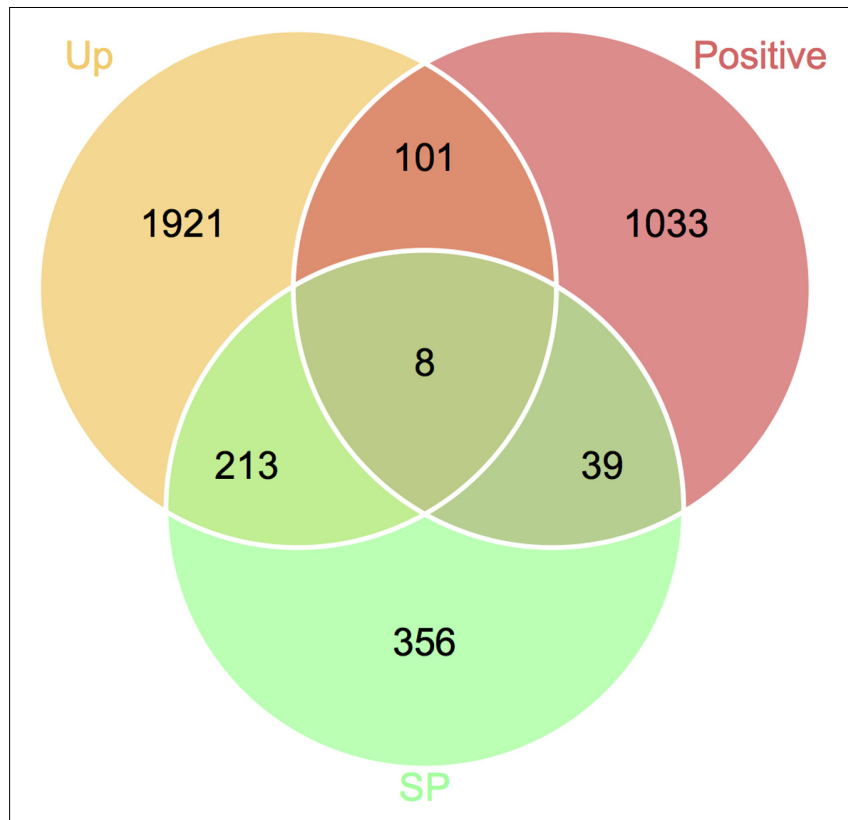

FIGURE 7 | Venn diagram showing the candidate genes responsible for Fusarium-wheat interaction. Genes that are up-regulated during plant infection (Up), under positive selection (Positive), and predicted to encode secreted proteins (SP) were used for screening.

by Bowtie 2 (Langmead and Salzberg, 2012) and variants were called by SAMtools (Li et al., 2009) with its default parameters. For Canadian strain FG1 and American strain FG2 (Walkowiak et al., 2015), the whole genome shotgun assembly (no raw data available) were used. To call precise variants, the contigs were reordered by ABACAS (Assefa et al., 2009) with the reference genome of strain PH-1 (King et al., 2015), and MUMmer (Delcher et al., 1999) were used to calling the variants with its default parameters.

For the phylogenomic analysis, all the SNP data were converted to segregating sites, and PhyML (Guindon et al., 2010) was used to build the maximum likelihood tree with its default parameters. The Venn diagram of SNPs and functionally lost genes was draw by InteractiVenn (Heberle et al., 2015). The annotation of variation was performed by snpEff (Cingolani et al., 2012). To visualize the genomic variation data, Circos (Krzywinski et al., 2009) were used. For the density calculation of variants, SP and GC contents, the entire genome was split to $25 \mathrm{~kb}$ intervals. To model the genomic variations and estimate the variation rates, mixtools (Benaglia et al., 2009) developed in $\mathrm{R}$ were used. The hidden Markov states of each interval were determined by using $\mathrm{R}$ package depmixS4 (Visser and Speekenbrink, 2010). The gene length, exon numbers, and FIRs analysis were extracted or calculated from the completed genome annotation (King et al., 2015) by custom Perl scripts. GO enrichment analysis was performed with Blast2GO (Conesa et al., 2005), and the $p$-values were adjusted with Benjamini-Hochberg procedure by controlling false discovery rate (FDR) to 0.05 . All the Perl, R, and Shell scripts used in this study for resequencing and other analysis were available on GitHub ${ }^{1}$.

\section{Positive Selection Analysis}

To evaluate the selection pressures across different strains of F. graminearum, each SNP in the genes were used to generate an alternative sequence in all the resequenced strains analyzed here. Since only SNP data were used, the alignment of the sequences is thus codon-aligned. The proportions of synonymous and nonsynonymous (Langmead et al., 2009) differences per sites (Nei and Gojobori, 1986) were calculated by SNAP (Ganeshan et al., 1997). Two different methods were used to screen the positive selected genes $(\mathrm{pN}>\mathrm{pS})$ from the 21 pairwise comparisons of the $\mathrm{pN}$ and $\mathrm{pS}$ values in the seven strains (PH-1, YL-1, HN9-1, HN-Z6, CS3005, FG1, and FG2). First, if a gene has a larger pN value than $\mathrm{pS}$ value in more than $50 \%$ of the total 21 pairwise comparisons (i.e., at least 11 pairwise), it is regarded as a positive selected gene. Second, if the average $\mathrm{pN}$ value of a gene is larger than its average $\mathrm{pS}$ value that is not 0 , it is also regarded as a positive selected gene.

\section{RNA-Seq Analysis}

For sampling, freshly harvested $\mathrm{PH}-1$ conidia from carboxymethyl cellulose cultures were re-suspended to a final concentration of $10^{5}$ spores per milliliter in sterile distilled

${ }^{1}$ https://github.com/wangqinhu/fgreseq

TABLE 4 | Candidate genes responsible for Fusarium-wheat interaction.

\begin{tabular}{lcccc}
\hline Gene ID & Length $(\mathbf{a a})$ & Subgenome & PHI phenotype & Annotation \\
\hline FGRRES_00006* & 296 & Fast & Reduced virulence & \\
FGRRES_00061 & 125 & Fast & & Gegh 16 protein \\
FGRRES_00184 & 314 & Fast & Unaffected pathogenicity & Killer kp4 \\
FGRRES_06733 & 716 & Fast & Reduced virulence & Catalase \\
FGRRES_10712 & 395 & Fast & Unaffected pathogenicity & Alkaline Protease \\
FGRRES_10713 & 557 & Fast & Reduced virulence & Unknown \\
FGRRES_13515*\# & 122 & Slow & Xylanase \\
FGRRES_15917_M+ & 276 & & Reduce
\end{tabular}

*Have loss-of-function variation found in resequenced strains. \#Identified as one of the pathogen-associated gene that described in Sperschneider et al. (2013). ${ }^{\$}$ Homologous to the effector candidate FGSG_03315 identified by interspecies genome comparison (Sperschneider et al., 2015). ${ }^{\dagger}$ Homologous to the effector candidate FGSG_10999 identified by interspecies genome comparison (Sperschneider et al., 2015). 
water. Flowering wheat heads of cultivar XiaoYan 22 were dropinoculated with $10 \mu \mathrm{l}$ of conidium suspensions as described previously (Jiang et al., 2015). Total RNAs were extracted with TRIzol (Invitrogen, USA) and treated with RNase-free DNase I. RNA-seq libraries with the average insert size of $300 \mathrm{bp}$ were constructed as described in the manufacture. Illumina deep sequencing with paired-end $2 \times 125$ bp model were performed at Novogene Bioinformatics Technology (Beijing, China). RNA-seq data were deposited at NCBI SRA database under accession number SRP063766. The mycelia RNA-seq data were downloaded from NCBI SRA database under accession number SRP060552, which were generated by our lab previously using the same protocol. The paired end clean reads (Supplementary Table S1) were mapped to the F. graminearum reference genome (Cuomo et al., 2007; King et al., 2015) by hisat (Kim et al., 2015) and the abundances of the gene expression were count by using featureCounts (Liao et al., 2014). The differences of gene expression between the infection tissue and mycelia with two biological repeats were calculated with $\mathrm{R}$ package edgeRun (Dimont et al., 2015). A gene with $\log _{2} \mathrm{FC}$ ( $\log _{2}$ fold change) greater than 1 and FDR less than 0.05 was regarded as DEGs.

\section{ChIP-Seq Analysis}

The F. graminearum ChIP-seq (chromatin immunoprecipitation and high throughput DNA sequencing) data (Connolly et al., 2013) of H3K4me2 (SRR999613, SRR999614, SRR999615, SRR999616), H3K4me3 (SRR999617, SRR999618), and H3K27me3 (SRR999608, SRR999609, SRR999610) from mycelia were downloaded from NCBI SRA database. They were mapped on strain PH-1 reference genome via Bowtie (Langmead et al., 2009). The mapped bam files from the high and low nitrogen conditions were merged with SAMtools (Li et al., 2009) and the coverage was calculated with BEDtools (Quinlan and Hall, 2010). For correlation analysis, Spearman correlation developed in $\mathrm{R}$ was used. The ChIP-seq and relevant RNA-seq data were visualized by Circos (Krzywinski et al., 2009).

\section{Pathogenicity and Adaption Related Genes}

For CAZY genes (Henrissat and Davies, 1997) in F. graminearum, Zhao et al. (2013) dataset without glycosyltransferases (irrelevant to pathogenicity) were used for analysis. F. graminearum genes have a significant hit (E-value cut-off $=1 \mathrm{e}-5)$ against $\mathrm{PHI}$ database (Urban et al., 2015) were defined as PHI genes. For PA genes, Sperschneider et al. (2013) datasets were used. The SM

\section{REFERENCES}

Assefa, S., Keane, T. M., Otto, T. D., Newbold, C., and Berriman, M. (2009). ABACAS: algorithm-based automatic contiguation of assembled sequences. Bioinformatics 25, 1968-1969. doi: 10.1093/bioinformatics/btp347

Benaglia, T., Chauveau, D., Hunter, D. R., and Young, D. S. (2009). mixtools: an R package for analyzing finite mixture models. J. Stat. Softw. 32, 1-29. doi: $10.18637 /$ jss.v032.106

Brown, N. A., Antoniw, J., and Hammond-Kosack, K. E. (2012). The predicted secretome of the plant pathogenic fungus Fusarium graminearum: a refined comparative analysis. PLoS ONE 7:e33731. doi: 10.1371/journal.pone.0033731 genes in F. graminearum were described by Sieber et al. (2014). For SP, the candidates developed in the completed genome of F. graminearum by Brown et al. (2012) algorithm were used. These SP have excluded the ones containing transmembrane domains or GPI anchors, and the subcellular localization was further evaluated by WolfPsort.

\section{Statistical Tests}

One-sided $t$-tests were applied to compare the difference of GC content, gene length, $\mathrm{pN}$, and gene up-regulation/downregulation in the fast and slow subgenomes. One-sided Fisher's exact tests were used to compare the numbers of SP, positive selection genes, up-regulated/down-regulated genes, and infection-related genes in the two-speed subgenomes. To access the variations difference of the exon number in the two-speed subgenomes, $F$-test was performed. All the statistical tests and related graphics were performed and illustrated with $\mathrm{R}$.

\section{AUTHOR CONTRIBUTIONS}

HL and J-RX conceived and designed the experiments; QW, HL, CJ, CC, and CW performed the analysis; QW, HL, and J-RX wrote the manuscript.

\section{FUNDING}

This work was supported by the Fundamental Research Funds for the Central Universities (2452015011 to HL) and grants from the Special Fund for Agro-scientific Research in the Public Interest (201303016 to for J-RX), and China Postdoctoral Science Foundation (2015M580884 to QW).

\section{ACKNOWLEDGMENT}

We thank Drs. Zhonghua Ma and Wenming Zheng for kindly providing the strains.

\section{SUPPLEMENTARY MATERIAL}

The Supplementary Material for this article can be found online at: http://journal.frontiersin.org/article/10.3389/fpls.2017.00140/ full\#supplementary-material

Cingolani, P., Platts, A., Wang le, L., Coon, M., Nguyen, T., Wang, L., et al. (2012). A program for annotating and predicting the effects of single nucleotide polymorphisms, SnpEff: SNPs in the genome of Drosophila melanogaster strain w1118; iso-2; iso-3. Fly (Austin) 6, 80-92. doi: 10.4161/fly. 19695

Conesa, A., Gotz, S., Garcia-Gomez, J. M., Terol, J., Talon, M., and Robles, M. (2005). Blast2GO: a universal tool for annotation, visualization and analysis in functional genomics research. Bioinformatics 21, 3674-3676. doi: 10.1093/ bioinformatics/bti610

Connolly, L. R., Smith, K. M., and Freitag, M. (2013). The Fusarium graminearum histone H3 K27 methyltransferase KMT6 regulates development 
and expression of secondary metabolite gene clusters. PLoS Genet. 9:e1003916. doi: 10.1371/journal.pgen.1003916

Cuomo, C. A., Guldener, U., Xu, J. R., Trail, F., Turgeon, B. G., Di Pietro, A., et al. (2007). The Fusarium graminearum genome reveals a link between localized polymorphism and pathogen specialization. Science 317, 1400-1402. doi: 10. 1126/science. 1143708

Delcher, A. L., Kasif, S., Fleischmann, R. D., Peterson, J., White, O., and Salzberg, S. L. (1999). Alignment of whole genomes. Nucleic Acids Res. 27, 2369-2376. doi: $10.1093 /$ nar/27.11.2369

Dimont, E., Shi, J., Kirchner, R., and Hide, W. (2015). edgeRun: an R package for sensitive, functionally relevant differential expression discovery using an unconditional exact test. Bioinformatics 31, 2589-2590. doi: 10.1093/ bioinformatics/btv209

D’Mello, J. P. F., Placinta, C. M., and Macdonald, A. M. C. (1999). Fusarium mycotoxins: a review of global implications for animal health, welfare and productivity. Anim. Feed Sci. Technol. 80, 183-205. doi: 10.1016/S03778401(99)00059-0

Dong, S., Raffaele, S., and Kamoun, S. (2015). The two-speed genomes of filamentous pathogens: waltz with plants. Curr. Opin. Genet. Dev. 35, 57-65. doi: 10.1016/j.gde.2015.09.001

Ganeshan, S., Dickover, R. E., Korber, B. T., Bryson, Y. J., and Wolinsky, S. M. (1997). Human immunodeficiency virus type 1 genetic evolution in children with different rates of development of disease. J. Virol. 71, 663-677.

Gardiner, D. M., Stiller, J., and Kazan, K. (2014). Genome sequence of Fusarium graminearum isolate CS3005. Genome Announc 2, e00227-14. doi: 10.1128/ genomeA.00227-14

Goswami, R. S., and Kistler, H. C. (2004). Heading for disaster: Fusarium graminearum on cereal crops. Mol. Plant Pathol. 5, 515-525. doi: 10.1111/j. 1364-3703.2004.00252.x

Grandaubert, J., Lowe, R. G., Soyer, J. L., Schoch, C. L., Van de Wouw, A. P., Fudal, I., et al. (2014). Transposable element-assisted evolution and adaptation to host plant within the Leptosphaeria maculans-Leptosphaeria biglobosa species complex of fungal pathogens. BMC Genomics 15:891. doi: 10.1186/1471-216415-891

Guindon, S., Dufayard, J. F., Lefort, V., Anisimova, M., Hordijk, W., and Gascuel, O. (2010). New algorithms and methods to estimate maximumlikelihood phylogenies: assessing the performance of PhyML 3.0. Syst. Biol. 59, 307-321. doi: 10.1093/sysbio/syq010

Haas, B. J., Kamoun, S., Zody, M. C., Jiang, R. H., Handsaker, R. E., Cano, L. M., et al. (2009). Genome sequence and analysis of the Irish potato famine pathogen Phytophthora infestans. Nature 461, 393-398. doi: 10.1038/nature 08358

Haggblom, P., and Nordkvist, E. (2015). Deoxynivalenol, zearalenone, and Fusarium graminearum contamination of cereal straw; field distribution; and sampling of big bales. Mycotoxin Res. 31, 101-107. doi: 10.1007/s12550-015$0220-\mathrm{z}$

Heberle, H., Meirelles, G. V., da Silva, F. R., Telles, G. P., and Minghim, R. (2015). InteractiVenn: a web-based tool for the analysis of sets through Venn diagrams. BMC Bioinformatics 16:169. doi: 10.1186/s12859-015-0611-3

Henrissat, B., and Davies, G. (1997). Structural and sequence-based classification of glycoside hydrolases. Curr. Opin. Struct. Biol. 7, 637-644. doi: 10.1016/S0959$440 \mathrm{X}(97) 80072-3$

Hussein, H. S., and Brasel, J. M. (2001). Toxicity, metabolism, and impact of mycotoxins on humans and animals. Toxicology 167, 101-134. doi: 10.1016/ S0300-483X(01)00471-1

Jiang, C., Zhang, S., Zhang, Q., Tao, Y., Wang, C., and Xu, J. R. (2015). FgSKN7 and FgATF1 have overlapping functions in ascosporogenesis, pathogenesis and stress responses in Fusarium graminearum. Environ. Microbiol. 17, 1245-1260. doi: 10.1111/1462-2920.12561

Kim, D., Langmead, B., and Salzberg, S. L. (2015). HISAT: a fast spliced aligner with low memory requirements. Nat. Methods 12, 357-360. doi: 10.1038/nmeth.3317

King, R., Urban, M., Hammond-Kosack, M. C., Hassani-Pak, K., and HammondKosack, K. E. (2015). The completed genome sequence of the pathogenic ascomycete fungus Fusarium graminearum. BMC Genomics 16:544. doi: 10. 1186/s12864-015-1756-1

Krzywinski, M., Schein, J., Birol, I., Connors, J., Gascoyne, R., Horsman, D., et al. (2009). Circos: an information aesthetic for comparative genomics. Genome Res. 19, 1639-1645. doi: 10.1101/gr.092759.109
Langmead, B., and Salzberg, S. L. (2012). Fast gapped-read alignment with bowtie 2. Nat. Methods 9, 357-359. doi: 10.1038/nmeth.1923

Langmead, B., Trapnell, C., Pop, M., and Salzberg, S. L. (2009). Ultrafast and memory-efficient alignment of short DNA sequences to the human genome. Genome Biol. 10:R25. doi: 10.1186/gb-2009-10-3-r25

Li, H., Handsaker, B., Wysoker, A., Fennell, T., Ruan, J., Homer, N., et al. (2009). The sequence alignment/map format and SAMtools. Bioinformatics 25, 20782079. doi: 10.1093/bioinformatics/btp352

Liao, Y., Smyth, G. K., and Shi, W. (2014). featureCounts: an efficient general purpose program for assigning sequence reads to genomic features. Bioinformatics 30, 923-930. doi: 10.1093/bioinformatics/btt656

Ma, L. J., van der Does, H. C., Borkovich, K. A., Coleman, J. J., Daboussi, M. J., Di Pietro, A., et al. (2010). Comparative genomics reveals mobile pathogenicity chromosomes in Fusarium. Nature 464, 367-373. doi: 10.1038/nature08850

Mehta, Y. (2014). "Spike diseases caused by fungi," in Wheat Diseases and Their Management, (Cham: Springer International Publishing), 65-104. Available at: http://link.springer.com/book/10.1007/978-3-319-06465-9

Nei, M., and Gojobori, T. (1986). Simple methods for estimating the numbers of synonymous and nonsynonymous nucleotide substitutions. Mol. Biol. Evol. 3, 418-426.

Qu, B., Li, H. P., Zhang, J. B., Xu, Y. B., Huang, T., Wu, A. B., et al. (2008). Geographic distribution and genetic diversity of Fusarium graminearum and F. asiaticum on wheat spikes throughout China. Plant Pathol. 57, 15-24. doi: 10.1111/j.1365-3059.2007.01711.x

Quinlan, A. R., and Hall, I. M. (2010). BEDTools: a flexible suite of utilities for comparing genomic features. Bioinformatics 26, 841-842. doi: 10.1093/ bioinformatics/btq033

Raffaele, S., Farrer, R. A., Cano, L. M., Studholme, D. J., MacLean, D., Thines, M., et al. (2010). Genome evolution following host jumps in the Irish potato famine pathogen lineage. Science 330, 1540-1543. doi: 10.1126/science.1193070

Reyes-Dominguez, Y., Boedi, S., Sulyok, M., Wiesenberger, G., Stoppacher, N., Krska, R., et al. (2012). Heterochromatin influences the secondary metabolite profile in the plant pathogen Fusarium graminearum. Fungal Genet. Biol. 49, 39-47. doi: 10.1016/j.fgb.2011.11.002

Sieber, C. M., Lee, W., Wong, P., Munsterkotter, M., Mewes, H. W., Schmeitzl, C., et al. (2014). The Fusarium graminearum genome reveals more secondary metabolite gene clusters and hints of horizontal gene transfer. PLoS ONE 9:e110311. doi: 10.1371/journal.pone.0110311

Soyer, J. L., El Ghalid, M., Glaser, N., Ollivier, B., Linglin, J., Grandaubert, J., et al. (2014). Epigenetic control of effector gene expression in the plant pathogenic fungus Leptosphaeria maculans. PLoS Genet. 10:e1004227. doi: 10.1371/journal. pgen.1004227

Soyer, J. L., Rouxel, T., and Fudal, I. (2015). Chromatin-based control of effector gene expression in plant-associated fungi. Curr. Opin. Plant Biol. 26, 51-56. doi: 10.1016/j.pbi.2015.05.025

Sperschneider, J., Gardiner, D. M., Taylor, J. M., Hane, J. K., Singh, K. B., and Manners, J. M. (2013). A comparative hidden Markov model analysis pipeline identifies proteins characteristic of cereal-infecting fungi. BMC Genomics 14:807. doi: 10.1186/1471-2164-14-807

Sperschneider, J., Gardiner, D. M., Thatcher, L. F., Lyons, R., Singh, K. B., Manners, J. M., et al. (2015). Genome-wide analysis in three Fusarium pathogens identifies rapidly evolving chromosomes and genes associated with pathogenicity. Genome Biol. Evol. 7, 1613-1627. doi: 10.1093/gbe/ evv092

Talas, F., and McDonald, B. A. (2015). Genome-wide analysis of Fusarium graminearum field populations reveals hotspots of recombination. BMC Genomics 16:996. doi: 10.1186/s12864-015-2166-0

Urban, M., Pant, R., Raghunath, A., Irvine, A. G., Pedro, H., and HammondKosack, K. E. (2015). The pathogen-host interactions database (PHI-base): additions and future developments. Nucleic Acids Res. 43, D645-D655. doi: $10.1093 /$ nar/gkul165

van der Lee, T., Zhang, H., van Diepeningen, A., and Waalwijk, C. (2015). Biogeography of Fusarium graminearum species complex and chemotypes: a review. Food Addit. Contam. Part A Chem. Anal. Control Expo. Risk Assess. 32, 453-460. doi: 10.1080/19440049.2014.984244

Visentin, I., Montis, V., Doll, K., Alabouvette, C., Tamietti, G., Karlovsky, P., et al. (2012). Transcription of genes in the biosynthetic pathway for fumonisin mycotoxins is epigenetically and differentially regulated in the fungal maize 
pathogen Fusarium verticillioides. Eukaryot. Cell 11, 252-259. doi: 10.1128/EC. 05159-11

Visser, I., and Speekenbrink, M. (2010). depmixS4: an R package for hidden Markov models. J. Statist. Softw. 36, 1-21. doi: 10.18637/jss.v036.i07

Walkowiak, S., Bonner, C. T., Wang, L., Blackwell, B., Rowland, O., and Subramaniam, R. (2015). Intraspecies interaction of Fusarium graminearum contributes to reduced toxin production and virulence. Mol. Plant Microbe Interact. 28, 1256-1267. doi: 10.1094/MPMI-06-15-0120-R

Yang, L., van der Lee, T., Yang, X., Yu, D., and Waalwijk, C. (2008). Fusarium populations on Chinese barley show a dramatic gradient in mycotoxin profiles. Phytopathology 98, 719-727. doi: 10.1094/PHYTO-98-6-0719

Zhang, H., Van der Lee, T., Waalwijk, C., Chen, W., Xu, J., Xu, J., et al. (2012). Population analysis of the Fusarium graminearum species complex from wheat in China show a shift to more aggressive isolates. PLoS ONE 7:e31722. doi: 10.1371/journal.pone.0031722

Zhao, C., Waalwijk, C., de Wit, P. J., Tang, D., and van der Lee, T. (2014). Relocation of genes generates non-conserved chromosomal segments in
Fusarium graminearum that show distinct and co-regulated gene expression patterns. BMC Genomics 15:191. doi: 10.1186/1471-2164-15-191

Zhao, Z., Liu, H., Wang, C., and Xu, J. R. (2013). Comparative analysis of fungal genomes reveals different plant cell wall degrading capacity in fungi. BMC Genomics 14:274. doi: 10.1186/1471-2164$14-274$

Conflict of Interest Statement: The authors declare that the research was conducted in the absence of any commercial or financial relationships that could be construed as a potential conflict of interest.

Copyright () 2017 Wang, Jiang, Wang, Chen, Xu and Liu. This is an open-access article distributed under the terms of the Creative Commons Attribution License (CC BY). The use, distribution or reproduction in other forums is permitted, provided the original author(s) or licensor are credited and that the original publication in this journal is cited, in accordance with accepted academic practice. No use, distribution or reproduction is permitted which does not comply with these terms. 